February 4, 2021

\title{
PCT Theorem, Wightman Axioms and Conformal Bootstrap
}

\author{
Jnanadeva Maharana ${ }^{1}$ \\ E-mail maharana@iopb.res.in \\ Institute of Physics \\ and \\ NISER \\ Bhubaneswar - 751005, India
}

\begin{abstract}
The axiomatic Wightman formulation for nonderivative conformal field theory is adopted to derive conformal bootstrap equation for the four point function. The equivalence between PCT theorem and weak local commutativity, due to Jost, play a very crucial role in axiomatic field theory. The theorem is suitably adopted for conformal field theory to derive the desired equations in CFT. We demonstrate that the two Wightman functions are analytic continuation of each other.
\end{abstract}

\footnotetext{
${ }^{1}$ Adjunct Professor, NISER, Bhubaneswar
} 
The PCT theorem is very profound. The proof of the theorem is based on axioms of local field theories. It is a fundamental probe for our basic understanding of microscopic physics. Pauli and Lüder $[1,2,3]$ presented the first proof of the theorem. However, at that juncture, possibility of parity violation in weak interaction was not yet proposed by Yang and Lee [4]. Parity nonconservation was subsequently observed experimentally. Thus, in the proof of the Pauli-Lüder theorem, the violations of disctrete symmetries, such as $\mathrm{P}, \mathrm{C}$, and $\mathrm{T}$, in field theories, had not been envisaged. Jost $[5,6]$ proved the PCT theorem from the axioms of local field theories whereas earlier proofs were based on Lagrangian field theories. The fundamental nature of Jost's proof is that weak local commutativity (WLC) at the Jost points is necessary and sufficient condition for PCT symmetry. This aspect will be elaborated in the sequel. One of the most important consequences of PCT theorem is that masses of particle and antiparticle be equal. The best experimental test comes from the $K^{0}-\bar{K}^{0}$ mass difference [7]. The limit is $-4.0 \times 10^{-19} \mathrm{GeV}<m_{K^{0}}-m_{\bar{K}^{0}}<4.0 \times 10^{-19} \mathrm{GeV}$. Moreover, the violation of PCT invariance of any Wightman function implies the violation of Lorentz invariance [8]. Therefore, there is so much of premium on the PCT theorem.

The purpose of this letter is to derive the conformal bootstrap equation in the Wightman's formulation of axiomatic field theory $[6,9]$. The PCT theorem is invoked to relate two four point Wightman functions. Our motivation is from following considerations. Let us envisage two four point Wightman functions: (i) $<0\left|\phi\left(x_{1}\right) \phi\left(x_{2}\right) \phi\left(x_{3}\right) \phi\left(x_{4}\right)\right| 0>$ and (ii) $<0\left|\phi\left(x_{4}\right) \phi\left(x_{3}\right) \phi\left(x_{2}\right) \phi\left(x_{1}\right)\right| 0>$. They are also boundary values of the analytically continued corresponding analytic functions of complexified coordinates as will be elaborated shortly. As such, there seems to be no relationship between them at this juncture; however, once PCT theorem is invoked they get related. As often the case in study of scattering amplitudes, seemingly unrelated amplitudes are no longer independent when we invoke a symmetry principle. For example the reactions, $\pi N \rightarrow \pi N$, are described by several independent amplitudes until we invoke the principle of isotpic spin invariance of strong interaction. Then the amplitudes $\pi^{+} p \rightarrow \pi^{+} p, \pi^{-} p \rightarrow \pi^{-} p$ and $\pi^{-} p \rightarrow \pi^{0} n$ are no longer independent. We appeal to PCT theorem, its equivalence to weak local commutativity and invoke the edge-of-the-wedge theorem to establish relation between the two Wightman functions, (i) and (ii) defined above. It will be shown that conformal bootstrap equation relating (i) and (ii) would be obtained. This is achieved by employing the conformal partial wave expansion procedure for each of the four point functions. It is assumed in our derivation that CPW converges for the case under consideration. We feel that it is a novel way to relate two different four point functions and obtain bootstrap equation. In order to derive the aforementioned result, we shall recapitulate important theorems of axiomatic field theory and recall some of the holomorphic properties of the Wightman functions [9]. We provide references to original papers and to books for the benefit of readers. The derivation of the conformal bootstrap 
equation is presented step by step sequentially starting from the important results of Wightman formulation. The rigorous results demonstrating intimate relationships between analyticity, crossing and causality in CFT and their connections with bootstrap will be presented in our forthcoming article [10].

The research activities in CFT sprouted following the seminal paper of Mack and Salam [11] and a lot of activties ensued in 1970's [12, 13, 14, 15]. Migdal introduced the idea of conformal invariance to derive bootstrap equations for hadronic interactions [16]. The conformal bootstrap was proposed by various authors in that period $[17,18,19]$. A rejuvinated activity has emerged in recent years and the conformal bootstrap program has expanded in several ditrections; these have been reviewed recently $[21,22,23]$. There has been vigorous research activities in CFT and it has spread in diverse directions such as the understanding of critical phenomena, supersymmetric conformal field theories in higher dimensions $(D>4)$. The studies of conformal properties of gauge theories and gravity has drawn a lot of attention [14]. One of the most exciting developments is the $A d S / C F T$ correspondance conjecture of Maldacena [24] which has strongly influenced research in supergravity and string theories.

The conformal transformation properties of a real scalar field, $\phi(x)$, are

$$
\begin{gathered}
{\left[P_{\mu}, \phi(x)\right]=i \partial_{\mu} \phi(x), \quad\left[M_{\mu \nu}, \phi(x)\right]=i\left(x_{\mu} \partial_{\nu}-x_{\nu} \partial_{\mu}\right) \phi(x)} \\
{[D, \phi(x)]=i\left(d+x^{\nu} \partial_{\nu}\right) \phi(x), \quad\left[K_{\mu}, \phi(x)\right]=i\left(x^{2} \partial_{\mu}-2 x_{\mu} x^{\nu} \partial_{\nu}-2 x_{\mu} d\right) \phi(x)}
\end{gathered}
$$

$\left\{P_{\mu}, M_{\mu \nu}\right\}$ are the ten generators of the Poincaré group; $D$ generates dilation and $K_{\mu}$ are the four special conformal transformation generators; $d$ is the scale dimension of $\phi(x)$.

Let us discuss some important features of conformal field theories. To begin with we recall that in the perturbative approach to field theory, crossing property of the scattering amplitude is maintained in each order. All Feynman diagrams, corresponding to direct channel and crossed channels, are included in every order. The analyticity and unitarity of amplitude are maintained according to stipulated rules in perturbation theoretic computations. In the context of phenomenological S-matrix theory, which described hadronic collisions, crossing is assumed. The idea of bootstrap evolved from the S-matrix philosophy. The bootstrap equations were used as consistency conditions in the S-matrix era. The rigorous proof of crossing for scattering amplitude was derived in subsequent years [25]. The bootstrap equations were introduced in conformal field theory in order to provide a rigorous basis to the phenomenological S-matrix notion of bootstrap. The structure of conformal field theories is deeply connected with the symmetry principles. In general, a Lagrangian density 
is not introduced, nor there is an action principle. Moreover, we do not invoke the concept of asymptotic fields and interacting fields. As a consequence, the axiomatic field theoretic techniques to compute scattering amplitude, for example in the LSZ formulation [26], turn out to be inadequate. Therefore, many of the rigorous results proved for scattering amplitudes from axiomatic field theories do not hold automatically in CFT. Important parameters are computed in CFT to test the theories against parameters of physical systems [23].

The correlation functions in CFT are of extreme importance. Furthermore, the analyticity and crossing properties play a crucial role in the study of CFT. Moreover, there is intimate relationship between causality and analyticity. Therefore, analyticity, crossing and causality are three ingredients in the study of CFT. It is natural to adopt Lorenzian metric. Our choice of the metric is $g_{\mu \nu}=\operatorname{diag}(+1,-1,-1,-1)$ and we work in four dimensional spacetime, $D=4$. We adopt Wighgtman's axiomatic formulation to investigate aforementioned attributes in CFT. The importance of Wightman function has been emphasized long ago in the intial developmental phase of CFT $[12,13,14,15,19,20]$. The axioms are $[9,39,6,40,41,42,43]$ :

(i) There exists a Hilbert space. It is constructed [14] with appropriate definitions for CFT.

(ii) The theory is conformally invariant and the vacuum, $\mid 0>$, is unique. It is annihilated by all the generators of the conformal group.

(iii) Spetrality: The energy and momentum of states are defined such that $0 \leq p^{2} \leq \infty$ and $p_{0} \geq 0$. We consider a class of theories such that the Fourier transform of the field, $\phi(x)$, i.e. $\tilde{\phi}(p)$, satisfies spectrality condition stated above [30, 14$]$.

(iv) Microcausality: Two local bosonic operators commute when their separation is spacelike i.e. $\left[\mathcal{O}(x), \mathcal{O}\left(x^{\prime}\right)\right]=0$; for $\left(x-x^{\prime}\right)^{2}<0$.

The operator product expansion (OPE) of Wilson [27] plays a crucial role in CFT. Consider product of two real scalar field operators $(A(x)$ is any real scalar field)

$$
A\left(x_{1}\right) A\left(x_{2}\right)=\sum f_{k}\left(x_{1}-x_{2}\right) C_{k}\left(\frac{x_{1}+x_{2}}{2}\right)
$$

This is a generic form of OPE. Here $\left\{f_{k}\left(x_{1}-x_{2}\right)\right\}$ are c-number functions which acquire singularities as $\left(x_{1}-x_{2}\right) \rightarrow 0$ and operators $\left\{C_{k}\right\}$ are local in $\left(x_{1}+x_{2}\right)$. The OPE, adopted for CFT, is very crucial. We remark that Wilson operator product expansion [27] was investigated from the Wighgtman axiom perspective by Wilson and Zimmerman [28]. Subsequently, Otterson and Zimmermann [29] advanced those technques.

A nonderivative scalar field $\phi(x)$, satisfying $\left[K_{\mu}, \phi(0)\right]=0, K_{\mu}$ being the generator of special conformal transformation, respects Wightman axioms [30]. The Fourier transform of $\phi(x), \tilde{\phi}(p)$, satisfies Wightman spectrality condition: $p \in V^{+}$, i.e. $p^{2} \geq 0$ 
and $p_{0} \geq 0$. Now on, $\phi(x)$ stands for a nonderivative real conformal field and any other generic scalar field is denoted as $A(x)$. We need to define the the structure of the Hilbert space. If we consider OPE of a pair of $\phi$ fields the form is

$$
\phi\left(x_{1}\right) \phi\left(x_{2}\right)=\sum_{n} \sum_{\chi} f_{n}^{\chi}\left(x_{1}-x_{2}\right) \mathcal{C}_{n}^{\chi}\left(\frac{x_{1}+x_{2}}{2}\right)
$$

Here $f_{n}^{\chi}$ are c-number coefficients which encode the short distance behaviour. $\mathcal{C}_{n}^{\chi}$ are composite fields belonging to the irreducible representations of $S U(2,2)$ which is the covering group of the conformal group $S O(4,2)$. For the case at hand, the set of fields $\left\{\mathcal{C}_{n}^{\chi}\right\}$ are of nonderivative type [30]. It has been proved by Mack [30] that the expansion is convergent, assuming that it is asymptotic one. Note that we need infinite number of such fields for the closure of the algebra [14]. The states are constructed by appealing to state $\leftrightarrow$ operator correspondences. Thus, we can identify state vectors of the underlying Hilbert space, $\mathcal{H}$. In view of preceding remarks, $\mathcal{H}$ decomposes into a direct sum of subspaces whose vectors belong to irreducible representations of $S U(2,2)$.

$$
\mathcal{H}=\oplus \mathcal{H}^{\chi}
$$

where $\chi$ collectively stands for all the quantum numbers that characterize an irreducible representation such as scale dimension, Lorentz spin etc. Therefore, two normalised vectors $\mid \chi_{i}>\in \mathcal{H}^{\chi_{i}}$ and $\mid \chi_{j}>\in \mathcal{H}^{\chi_{j}}$ satisfy $<\chi_{i} \mid \chi_{j}>=\delta_{i j}$.

We recapitulate the essential properties of the n-point Wightman functions, $W_{n}\left(x_{1}, x_{2}, \ldots x_{n}\right)$, defined to be

$$
W_{n}\left(x_{1}, x_{2}, \ldots x_{n}\right)=<0\left|\phi\left(x_{1}\right) \phi\left(x_{2}\right) \ldots \phi\left(x_{n}\right)\right| 0>
$$

in order to eventually derive the bootstrap equations. Note that $W_{n}\left(x_{1}, x_{2}, \ldots x_{n}\right)$ are not ordinary functions but are distributions. They are defined as

$$
W_{n}[f]=\int d^{4} x_{1} . . d^{4} x_{n} W\left(x_{1}, x_{2}, \ldots x_{n}\right) f\left(x_{1}, x_{2} \ldots x_{n}\right)
$$

These are linear functionals; consequently, a complex number is assigned with the introduction of $\left\{f\left(x_{1}, x_{2} . . x_{n}\right)\right\}$, the Schwarzian-type functions. $f\left(x_{1}, x_{2} . . x_{n}\right)$ is infinitely differentiable function with desired support properties in the spacetime manifold. Thus operators of the form $\phi[f]=\int d^{4} x \phi(x) f(x)$ are well defined. The npoint Wightman function is a distribution in the light of preceding remarks. Thus whenever we allude to the properties of $W_{n}\left(x_{1}, x_{2}, \ldots x_{n}\right)$, it is to be kept in mind that they are distributions; therefore, statements like convergence, limits etc. are to be understood in this context. It follows from translational invariance that $W_{n}$ depends on difference of coordinates: $W_{n}\left(x_{1}, x_{2}, \ldots x_{n}\right)=W_{n}\left(y_{1}, y_{2} \ldots . y_{n-1}\right)$, where 
$y_{j}=y_{j}-y_{j+1}$. Furthermore, $W_{n}\left(\left\{y_{j}\right\}\right)$ are invariant under Lorentz transformations: $W_{n}\left(y_{1}, \ldots y_{n-1}\right)=W_{n}\left(\Lambda_{r} y_{1}, \ldots \Lambda_{r} y_{n-1}\right)$ where $\Lambda_{r}$ is a real proper Lorentz transformation; det $\Lambda_{r}=1$. That physical momentum states are defined for lightlike momenta i.e. $p^{2} \geq 0$ and $p_{0} \geq 0$, implies that the Fourier transforms of $W_{n}\left(\left\{y_{j}\right\}\right), \widetilde{W}\left(p_{1}, \ldots p_{n-1}\right)=0$, unless $\left\{p_{j}\right\} \in V^{+}$. Define complex valued function $\mathcal{W}_{n}\left(\left\{\xi_{j}\right\}\right), j=1,2, . . n-1$. These complex variables are defined as $\xi_{j}^{\mu}=y_{j}^{\mu}-i \eta_{j}^{\mu}$; with the restrictions on the real set $\left(\left\{y_{j}, \eta_{j}\right\}\right)$, such that $\eta_{j}^{\mu} \in V^{+}$, and $-\infty<y_{i}^{\mu}<+\infty$; defining a forward tube, $T_{n-1}$. The distributions, $W_{n}\left(\left\{y_{j}\right\}\right)$, are boundary values of the analytic functions

$$
W_{n}\left(y_{1}, y_{2} \ldots y_{n-1}\right)=\lim _{\left\{\eta_{j}\right\} \rightarrow 0} \mathcal{W}_{n}\left(\xi_{1}, \xi_{2}, \ldots \xi_{n-1}\right)
$$

Notice that $\mathcal{W}_{n}\left(\left\{\xi_{j}\right\}\right)$ are invariant under real Lorentz transformations. The points, $\left\{\xi_{j}\right\} \in T_{n-1}$, generate a new set of points $\Lambda \xi_{1}, \Lambda \xi_{2}, \ldots \Lambda \xi_{n-1}$ under arbitrary complex Lorentz transformations where $\Lambda \in S L_{+}(2 \mathbf{C})$, det $\Lambda=1$. Thus the operation of $\Lambda$ on points of $T_{n-1}$ generates a new set of points which defines the extended tube $T_{n-1}^{\prime}$. Furthermore, the complex valued analytic function, $W_{n}(\{\xi\})$, is invariant under $S L_{+}(2 \mathbf{C})$ and possesses a single values continuation to $T_{n-1}^{\prime}[49]$. Note the important difference between points lying in $T_{n-1}$ and those lying in $T_{n-1}^{\prime}$ : the real points $\left\{y_{j}\right\}$ do not belong to the tube $T_{n-1}$ whereas $T_{n-1}^{\prime}$ contains the real points $\left\{y_{j}\right\}$. Where do these real points lie? Jost [5] proved an important theorem. The Jost points are spacetime points in which all convex combinations of successive differences are spacelike. For $\mathcal{W}_{n}\left(\xi_{1}, \xi_{2}, \ldots \xi_{n-1}\right)$ a Jost point is an ordered set $\left(x_{1}, x_{2} \ldots, x_{n}\right)$. The Jost theorem states [5]: A real point of $\left\{\xi_{1}, \xi_{2}, . . \xi_{n-1}\right\}$ lies in the extended tube, $T_{n-1}^{\prime}$, if and only if all real four vectors of the form $\sum_{1}^{n-1} \lambda_{j} \xi_{j}^{\mu}, \lambda_{j} \geq 0, \sum_{1}^{n-1} \lambda_{j}>0$ are spacelike i.e. $\left(\sum_{1}^{n-1} \lambda_{j} \xi_{j}^{\mu}\right)^{2}<0, \lambda_{j} \geq 0, \quad \sum_{1}^{n-1} \lambda_{j}>0$. The necessary and sufficient condition is that all the real points of $T_{n-1}^{\prime}$ are spacelike.

Recently, there have been considerable activities to investigate analyticity properties in CFT with Lorentzian signature metric [31, 32, 33, 34, 35, 36, 37]. We recall that there exists a close relationship between analyticity and crossing. Let us consider a four point Wightman function: $W_{4}\left(x_{1}, x_{2}, x_{3}, x_{4}\right)=<0\left|\phi\left(x_{1}\right) \phi\left(x_{2}\right) \phi\left(x_{3}\right) \phi\left(x_{4}\right)\right| 0>$ and the three permutated ones. They are equal

$$
\begin{aligned}
<0\left|\phi\left(x_{1}\right) \phi\left(x_{2}\right) \phi\left(x_{3}\right) \phi\left(x_{4}\right)\right| 0>=\quad & <0\left|\phi\left(x_{1}\right) \phi\left(x_{3}\right) \phi\left(x_{2}\right) \phi\left(x_{4}\right)\right| 0> \\
& =<0\left|\phi\left(x_{1}\right) \phi\left(x_{2}\right) \phi\left(x_{4}\right) \phi\left(x_{3}\right)\right| 0>
\end{aligned}
$$

when $\left(x_{2}-x_{3}\right)^{2}<0$ and $\left(x_{3}-x_{4}\right)^{2}<0$ since $\left.\left[\phi\left(x_{2}\right), \phi_{(} x_{3}\right)\right]=0$ and $\left[\phi\left(x_{3}\right), \phi\left(x_{4}\right)\right]=0$ due to microcausality. It should be borne in mind that the three Wightman functions are boundary values of analytic function, as we discuss later, and they coincide in the domain mentioned above. The three Wightman functions $W_{4}\left(x_{1}, x_{2}, x_{3}, x_{4}\right)$, $W_{4}\left(x_{1}, x_{3}, x_{2}, x_{4}\right)$ and $W_{4}\left(x_{1}, x_{2}, x_{4}, x_{3}\right)$ would be analytically continued to their corresponding tubes and extended tubes. When we look at their Fourier transforms, the 
support properties will be identified. We have proposed a method of analytic completion when a pair of permuted Wightman functions are considered at a time. In this light we have investigated crossing and analyticity of three point and four point functions in the Wightman formulation of CFT [38, 10]. In view of above remarks we should exercise caution while discussing analyticity and hence the crossing. We proceed to present how the conformal bootstrap equation follow from the above considerations.

Consider the first pair of correlators, (9), (i.e. where location of $\phi\left(x_{2}\right) \leftrightarrow \phi\left(x_{3}\right)$ are interchanged). If we adopt the conformal partial wave technique (CPW) $[48,19,21$, $22,23]$, on each side of the equation we obtain the desired bootstrap equation when $\left(x_{2}-x_{3}\right)^{2}<0$. We recall that, as always, one has to identify the domain where OPE converges in order to implement CPW expansion. Note, however, that it is desirable to prove that the two permuted Wightman functions are boundary values of analytic functions which coincide for spacelike separations of real coordinates. Thus the goal is to identify the domain of holomorphies of the two analytic functions. This is precisely accomplished in the proof of dispersion relations in axiomatic QFT [39]. The s and $u$ channel absorptive parts coincide in a spacelike separated region and then one proves that they are analytic continuation of each other [39]. We shall accomplish the task of analytic continuation presently. This proof of analyticity, to our knowledge is not comprehensively investigated for conformal field theories. We appeal to PCT theorem and its equivalence with WLC $[5,50]$ in order to derive the bootstrap condition in a domain of holomorphy as has been investigated in [10].

The PCT theorem in CFT: $W_{n}\left(x_{1}, x_{2} \ldots x_{n}\right)$, under PCT, transforms as

$$
W_{n}\left(\phi\left(x_{1}\right), \phi\left(x_{2}\right) \ldots \phi\left(x_{n}\right)\right) \rightarrow W_{n}\left(\phi\left(-x_{n}\right), \phi\left(-x_{n-1}\right), \ldots \phi\left(-x_{1}\right)\right)
$$

The PCT invariance of the theory implies

$$
<0\left|\phi\left(x_{1}\right) \phi\left(x_{2}\right) \ldots \phi\left(x_{n}\right)\right| 0>=<0\left|\phi\left(-x_{n}\right) \phi\left(x_{n-1}\right) \ldots \phi\left(-x_{1}\right)\right| 0>
$$

If the PCT theorem holds then for every $x_{1}, x_{2}, \ldots x_{n}$ with each $y_{j}=x_{j}-x_{j+1}$, a Jost point; the WLC condition implies

$$
<0\left|\phi\left(x_{1}\right) \phi\left(x_{2}\right) \ldots \phi\left(x_{n}\right)\right| 0>=<0\left|\phi\left(x_{n}\right) \phi\left(x_{n-1}\right) \ldots \phi\left(x_{1}\right)\right| 0>
$$

is satisfied.

We focus only on the four point function. There is a converse statement to Jost's theorem: if WLC holds in a real neighbourhood of (12), a Jost point, then the PCT condition (11) is valid everywhere. Note that WLC implies validity of PCT symmetry for the conformal scalar. We go through the following essential steps and 
refer to [10] for detailed expositions. The WLC theorem of Jost will be employed for $W_{4}\left(x_{1}, x_{2}, x_{3}, x_{4}\right)$ in what follows. As a consequence, of the WLC

$$
W_{4}\left(x_{1}, x_{2}, x_{3}, x_{4}\right)=W_{4}\left(x_{4}, x_{3}, x_{2}, x_{1}\right)
$$

The steps:

Step 1. Assume that CPT theorem is valid for the conformal theory. Recall that $\mathcal{W}_{4}\left(\xi_{1}, \xi_{2}, \xi_{3}\right)$ is a holomorphic function and (12) holds, for $n=4$, in the extended tube $T_{3}^{\prime}$ and the four point function is a boundary value of $\mathcal{W}_{4}\left(\xi_{1}, \xi_{2}, \xi_{3}\right)$,

$$
\lim _{\left\{\eta_{j}\right\} \rightarrow 0} \mathcal{W}_{4}\left(\xi_{1}, \xi_{2}, \xi_{3}\right)=W_{4}\left(y_{1}, y_{2}, y_{3}\right)
$$

Moreover, $\mathcal{W}_{4}\left(\xi_{1}, \xi_{2}, \xi_{3}\right)$ is invariant under proper complex Lorentz transformations, $S L_{+}(2 \mathbf{C}):\left\{\xi_{i}\right\} \rightarrow \Lambda\left\{\xi_{i}\right\}, \xi_{i} \in T_{3}^{\prime}$. Choose a $\Lambda$ such that the four complex vector $\xi_{i}^{\mu} \rightarrow-\xi_{i}^{\mu}, i=1,2,3$. Consequently,

$$
\mathcal{W}_{4}\left(\xi_{1}, \xi_{2}, \xi_{3}\right)=\mathcal{W}_{4}\left(-\xi_{1},-\xi_{2},-\xi_{3}\right)
$$

Step 2. Note that the r.h.s. of (11), for $n=4$, is also boundary value of an analytic function.

$$
\lim _{\left\{\eta_{j}\right\} \rightarrow 0} \mathcal{W}_{4}\left(\xi_{3}, \xi_{2}, \xi_{1}\right)=W_{4}\left(y_{3}, y_{2}, y_{1}\right)=<0\left|\phi\left(-x_{4}\right) \phi\left(-x_{3}\right) \phi\left(-x_{2}\right) \phi\left(-x_{1}\right)\right| 0>
$$

Step 3. Consider the difference of two 4-point functions: $\mathcal{W}_{4}\left(\xi_{1}, \xi_{2}, \xi_{3}\right)-\mathcal{W}_{4}\left(\xi_{3}, \xi_{2}, \xi_{1}\right)$. This is holomorphic in the domain $T_{3}^{\prime}$. This difference vanishes for $\operatorname{Re} \xi_{i}, i=1,2,3$ by CPT theorem (11). Now appeal to the edge-of-the-wedge theorem [51, 52] and we conclude

$$
\mathcal{W}_{4}\left(\xi_{1}, \xi_{2}, \xi_{3}\right)=\mathcal{W}_{4}\left(\xi_{3}, \xi_{2}, \xi_{1}\right)
$$

Consider the converse of this statement. Following Hall and Wightman [49], if (17) holds good in an arbitrary neighbourhood of $T_{3}^{\prime}$ it also holds good in the extended tube. Moreover, if it is also valid for passing into the boundary in the tube $T_{3}$ then we recover the condition of PCT invariance (11). Thus we conclude PCT invariance is equivalent to WLC in CFT. If we utilize the equations (15) and (17) then

$$
\mathcal{W}_{4}\left(\xi_{1}, \xi_{2}, \xi_{3}\right)=\mathcal{W}_{4}\left(-\xi_{1},-\xi_{2},-\xi_{3}\right)
$$

Remark: Suppose we try to pass to the boundary in the above equation for any set of $\left\{y_{i}\right\}$ in (18). We encounter the following problem. We shall not be able to get a relationship between the two function, in the above equation at these arbitrary real points. The reason is as $\xi_{1}, \xi_{2}, \xi_{3}$ approach real points the real vectors are in $V^{+}$; whereas the real vectors of $-\xi_{1},-\xi_{2},-\xi_{3}$ would be in $V^{-}$. Note the important inference: at the real point of holomorphy, this is the Jost point. Therefore, we have the equation

$$
\begin{aligned}
W_{4}\left(\xi_{1}, \xi_{2}, \xi_{3}\right)= & <0\left|\phi\left(x_{1}\right) \phi\left(x_{2}\right) \phi\left(x_{3}\right) \phi\left(x_{4}\right)\right| 0> \\
& =W\left(-\xi_{3},-\xi_{2},-\xi_{1}\right)=<0\left|\phi\left(x_{4}\right) \phi\left(x_{3}\right) \phi\left(x_{2}\right) \phi\left(x_{1}\right)\right| 0>
\end{aligned}
$$


This equation has important implication for the bootstrap equation as we shall demonstrate presently.

Let us consider $<0\left|\phi\left(x_{1}\right) \phi\left(x_{2}\right) \phi\left(x_{3}\right) \phi\left(x_{4}\right)\right| 0>$. We assume that conformal partial wave expansion is convergent for the case at hand. Then employ the conformal partial wave expansion by introducing a complete set of states, $\{|\Psi\rangle\}$, between the product of two pairs of operators: $\phi\left(x_{1}\right) \phi\left(x_{2}\right)$ and $\phi\left(x_{3}\right) \phi\left(x_{4}\right)$. These states, $\mid \Psi>$, span all the vectors of the Hilbert space, $\mathcal{H}$, i.e. all irreducible representations of the conformal group. The resulting equation is a familiar expression [23]

$W_{4}=<0\left|\phi\left(x_{1}\right) \phi\left(x_{2}\right) \phi\left(x_{3}\right) \phi\left(x_{4}\right)\right| 0>=\sum_{\mid \Psi>}<0\left|\phi\left(x_{1}\right) \phi\left(x_{2}\right)\right| \Psi><\Psi\left|\phi\left(x_{3}\right) \phi\left(x_{4}\right)\right| 0>(20)$

Now invoke state $\leftrightarrow$ operator correspondence and interprete $<0\left|\phi\left(x_{1}\right) \phi\left(x_{2}\right)\right| \Psi>$ as a three point function: $<0\left|\phi\left(x_{1}\right) \phi\left(x_{2}\right) \hat{\Psi}\right| 0>$ with the identification $|\Psi>=\hat{\Psi}| 0>$; $\hat{\Psi}$ represents the complete set of operator belonging to irreducible representations of the covering group. The second matrix element on the r.h.s of (20) becomes another three point function where $<\Psi|=<0| \hat{\bar{\Psi}} ; \hat{\bar{\Psi}}$ being the adjoint of $\hat{\Psi}$. Thus we express (20) as

$$
<0\left|\phi\left(x_{1}\right) \phi\left(x_{2}\right) \phi\left(x_{3}\right) \phi\left(x_{4}\right)\right| 0>=\sum_{\hat{\Psi}} \sum_{\alpha \beta} \lambda_{\phi_{1} \phi_{2}}^{\alpha} \mathcal{W}_{\phi_{1} \phi_{2} \hat{\Psi} \bar{\Psi} \phi_{3} \phi_{4}}^{\alpha \beta} \lambda_{\phi_{3} \phi_{4}}^{\beta}
$$

now $\lambda_{\phi_{1} \phi_{2}}^{\alpha}$ and $\lambda_{\phi_{3} \phi_{4}}^{\beta}$ can be read off from the above equation. Furthermore, $\mathcal{W}_{\phi_{1} \phi_{2} \hat{\Psi} \bar{\Psi} \phi_{3} \phi_{4}}^{\alpha \beta}$ is the conformal partial waves (CPW) $[48,21,22,23]$. The above equation is to be understood in the sense that it holds in a domain where the CPW expansion converges. The four point Wightman function appearing on the l.h.s. of (21) is boundary value of an analytic function in $T_{3}^{\prime}$.

Now focus on the CPW expansion

$$
<0\left|\phi\left(x_{4}\right) \phi\left(x_{3}\right) \phi\left(x_{2}\right) \phi\left(x_{1}\right)\right| 0>=\sum_{\hat{\Psi}, \bar{\Psi}} \sum_{\alpha \beta} \lambda_{\phi_{4} \phi_{3}}^{\alpha} \mathcal{W}_{\phi_{4} \phi_{3} \hat{\Psi} \bar{\Psi} \phi_{2} \phi_{1}}^{\alpha \beta} \lambda_{\phi_{2} \phi_{1}}^{\beta}
$$

Recall the equivalence theorem relating the PCT theorem and WLC $[5,50]$. The two expressions for Wightman functions (21) and (22) are equal at those Jost points and where conformal bootstrap conditions $[48,19,21,22,23]$ are valid.

It follows from our sequence of arguments that these two functions are analytic functions in extended tubes. Thus by invoking Jost's theorem and Dyson's [50] proof of analyticity it follows that the two equations (21) and (22) are analytic continuations of each other since (19) holds at Jost points. The bootstrap relation

$$
\sum_{\hat{\Psi}} \sum_{\alpha \beta} \lambda_{\phi_{1} \phi_{2}}^{\alpha} \mathcal{W}_{\phi_{1} \phi_{2} \hat{\Psi} \bar{\Psi} \phi_{3} \phi_{4}}^{\alpha \beta} \lambda_{\phi_{3} \phi_{4}}^{\beta}=\sum_{\hat{\Psi}, \hat{\Psi}} \sum_{\alpha \beta} \lambda_{\phi_{4} \phi_{3}}^{\alpha} \mathcal{W}_{\phi_{4} \phi_{3} \hat{\Psi} \bar{\Psi} \phi_{2} \phi_{1}}^{\alpha \beta} \lambda_{\phi_{2} \phi_{1}}^{\beta}
$$

is an equation involving spacetime coordinates satisfying Jost's condition. It is quite remarkable that using the power of PCT theorem and WLC together with the analyticity properties alluded to above, it is possible to demonstrate that bootstrap relation 
holds since the two four point functions are analytic continuation of each other (by edge-of-wedge theorem). Notice that if we permute a given Wightman function to obtain another one then the pair are analytic continuation of each other. We have proved in the forthcoming longer paper [10] that a pair of four point Wightman functions are analytic in the unions of their domains of holomorphy i.e. the union of the corresponding extended tubes, $T_{3}^{\prime}$ 's. Consider, as an example, a permuted four point function: $<0\left|\phi\left(x_{1}\right) \phi\left(x_{2}\right) \phi\left(x_{4}\right) \phi\left(x_{3}\right)\right| 0>$. It has been shown by us that these two four point functions are analytic functions in their corresponding extended tubes. Moreover, for $\left(x_{3}-x_{4}\right)^{2}<0$ they coincide. Then one argues that the two are analytic continuation of each other. The conformal bootstrap equation can be obtained modulo identifying the domain of convergence of the OPE involved.

Remarks: The above bootstrap condition is not obtained specifically for a scalar conformal field theory. If we consider four point Wightman function for nonderivative conformal fields which belong to irreducible representation of conformal group then the above proof will go through with appropriate modifications. Now the corresponding Wightman function will carry tensor indices as the fields would transform according to the representations of $S L(2 \mathbf{C}) \otimes S L(2 \mathbf{C})$ and they will carry their conformal dimensions [14]. Therefore, the four point function will have a tensor structure inherited from the product of four field operators, each field belonging to representation of $S L(2 \mathbf{C}) \otimes S L(2 \mathbf{C})$ in a general setting. Thus the $W_{4}$ will be decopmosed accordingly and transform covariantly under representations of $S L(2 \mathbf{C}) \otimes S L(2 \mathbf{C})$. The preceding arguments will essentially go through. Consequently, the analyticity properties and bootstrap equations will continue to hold. Therefore, we conclude that the two resulting four point functions will be analytic continuation of each other.

Conclusions. We have rigorously derived that the conformal bootstrap equations hold, for conformal scalar field $\phi(x)$, in the extended tube $T_{3}^{\prime}$, for the four point function. The power of the WLC is crucial to prove this bootstrap condition. The proof is based on Wightman axioms for CFT, $\phi(x)$. We have argued that conformal bootstrap conditions will also hold for four point functions of conformal fields belonging irreducible representations of conformal group so long as they are of nonderivative type fields. The work is in progress to study analyticity and crossing for n-point functions in conformal field theories in the present optics.

Acknowledgments: I am indebted to Ba-Gaga for their love, affections, patience and for my raison d'être. 


\section{References}

1. W. Pauli, in Niels Bohr and the Development of Physics, McGraw-Hill, New York (1955) pp 30.

2. G. Lüders, Danske Videnskabernes Selskab, Mat.-fys. Medd. 28, No 5 (1954).

3. G. Grawert, G. Lüders and H. Rollnik, Fortscr. der Physik, 7, 291 (1959).

4. C. N. Yang and T. D. Lee, Phys. Rev., 104, 254 (1956).

5. R. Jost, Helv. Phys. Acta, 30, 409 (1957).

6. R. F. Streater and A. S. Wightman, PCT, Spin and Statistics, and All That, W. A. Benjamin, Inc. New York Amsterdam, 1964.

7. P. A. Zyla et al.(Particle Data Group), Prog. Th. Phys. 2020, 083 C01 (2020).

8. O. W. Greenberg, Phys. Rev. Lett. 89, 231602 (2002).

9. A. S. Wightman, Phys. Rev. 101, 860 (1956).

10. J. Maharana, Crossing, Causality and Analyticity in Conformal Field Theory, to be submitted for publication.

11. G. Mack and A. Salam, Ann. Phys. 53 , 174 ( 1969).

12. S. Ferrara, R. Gatto and A. F. Grillo, Springer Tracts in Mod. Phys. 67, 1 (1973).

13. E. S. Fradkin and M. Ya Palchik, Phys. Rep. C44, 249 (1978).

14. E. S. Fradkin and M. Ya Palchik, Conformal Field Theory in D-dimensions, Springer Science Business Media, Dordrecht, 1996.

15. I. T. Todorov, M. C. Mintechev and V.R. Petkova, Conformal Invariance in Quantum Field Theory, Publications of Scuola Normale Superiore, Birkhäuser Verlag, 2007.

16. A. A. Migdal, Phys. Lett. 37B, 98 (1971); Phys. Lett. 37B, 386 (1971).

17. S. Ferrara, A. F. Grillo and R. Gatto, Annals of Phys. 76, 161 (1973).

18. S. Ferrara, A. F. Grillo, R. Gatto and G. Parisi, Nuovo. Cim. A19, 667 (1974).

19. A. M. Polyakov, Z. Eksp. Teor. Fiz, 66, 23 (1974).

20. M. Lüscher and G. Mack, Commun. Math. Phys. 41, 203 (1975). 
21. D. Simon-Duffin, TASI Lectures 2015, arXiv: 1602.07982[hep-th].

22. J. Penedones, TASI Lecture 2016, arXiv: 1608.04948[hep-th].

23. D. Poland, S. Rychkov and A. Vichi, Rev. Mod. Phys. 91, 051002 (2019).

24. J. Maldacena, Adv. Theor. Math. Phys. 2, 231 (1998).

25. J. Bross, H. Epstein and V. Glaser, Nuovo. Cim. 31, 1265 (1964).

26. H. Lehmann, K. Symanzik and W. Zimmermann, Nuovo. Cim. 1, 201 (1955).

27. K. G. Wilson, Phys. Rev. 179, 1499 (1969).

28. K. G. Wilson and W. Zimmermann, Commun. Math. Phys. 24, 87 (1972).

29. P. Otterson and W. Zimmermann, Commun. Math. Phys. 24, 107 (1972).

30. G. Mack, Commun. Math. Phys. 53, 155 (1977).

31. Z. Komargodski and A. Zhiboedov JHEP, 11, 140 (2013).

32. T. Hartman, S. Kundu and A. Tajdini, JHEP 07, 066 (2017).

33. T. Hartman, S. Jain and S. Kundu, JHEP 05, 099 (2016).

34. M. S. Costa, T. Hansen and J. Penedones, JHEP 10, 197 (2017).

35. S. Caron-Huot, JHEP 09, 078 (2017).

36. T. Baurista and H. Godazgar, JHEP 01, 142 (2020).

37. M. Gillioz, Commun. Math. Phys. 379, 227 (2020).

38. J. Maharana, Mod. Phys. Lett. A35, 2050186, (2020).

39. S. S. Schweber, Introduction to Relativistic Quantum Field Theory, Harper and Row, New York, Evaston and London, 1961.

40. R. Jost, General Theory of Quantized Fields, American Mathematical Society, Providence, Rhode Island, 1965.

41. R. Haag, Local Quantum Physics: Fields, Particles, Algebras, Springer, 1996.

42. C. Itzykson and J. -B. Zubber Quantum Field Theory, Dover Publications Mineola, New York, 2008.

43. N. N. Bogolibov, A.A. Logunov, A.I. Oksak and I. T. Todorov, General Principles of Quantum Field Theory, Klwer Academic Publisher, Dordrecht/Boston/New York/London, 1990. 
44. T. Yao, J. Math. Phys. 8, 1731 (1967).

45. T. Yao, J. Math. Phys. 9, 1615 (1968).

46. T. Yao, J. Math. Phys. 12, 315 (1971).

47. G. Mack, Commun. Math. Phys. 55, 1 (1972).

48. S. Ferrara, A. F. Grillo and R. Gatto, Lett. Nuovo, Cimento, 2, 1363 (1971).

49. D. Hall and A. S. Wightman, Mat. Fys. Medd. Dan. Vid. Selsk., 31, 5 (1957).

50. F. J. Dyson, Phys. Rev. 110, 579 (1958).

51. H. J. Bremmermann, R. Oehme and J. G. Taylor, Phys. Rev., 109, 2178 (1958). The proof was for pion-nucleon scattering in the LSZ formalism.

52. H. Epstein, J. Math. Phys., 1, 524 (1963). 